\title{
Forensic pregnancy diagnostics with placental mRNA markers
}

\author{
Jeanot Gauvin • Dmitry Zubakov • \\ Joke van Rhee-Binkhorst • Ate Kloosterman • \\ Eric Steegers • Manfred Kayser
}

Received: 29 September 2008 / Accepted: 22 December 2008 / Published online: 16 January 2009

(C) The Author(s) 2009. This article is published with open access at Springerlink.com

\begin{abstract}
Current methods for pregnancy diagnostics are based on immunodetection of pregnancy-specific proteins and in a forensic context suffer from sensitivity and specificity issues. Here, we applied reverse transcriptase polymerase chain reaction (RT-PCR) technology to 11 genes previously reported with placental mRNA circulating in maternal blood. We found two genes, $h P L$ and $\beta h C G$, with pregnancy-specific expression in whole blood samples. RT-PCR detection of $h P L$ was positive in all samples tested throughout the pregnancy, whereas $\beta h C G$ was detectable until half of the second trimester but not at later gestation ages. For $h P L$, in vitro stability of the transcript was
\end{abstract}

Jeanot Gauvin, Dmitry Zubakov contributed equally to this work.

Electronic supplementary material The online version of this article (doi:10.1007/s00414-008-0315-6) contains supplementary material, which is available to authorized users.

J. Gauvin · D. Zubakov • M. Kayser $(\bowtie)$

Department of Forensic Molecular Biology,

Erasmus University Medical Center Rotterdam, P.O. Box 2040, 3000 CA Rotterdam, The Netherlands

e-mail: m.kayser@erasmusmc.nl

J. Gauvin · A. Kloosterman

Master in Forensic Science Program,

Amsterdam Professional School of Science,

University of Amsterdam,

Amsterdam, The Netherlands

J. van Rhee-Binkhorst • E. Steegers

Department of Obstetrics and Gynecology,

Division of Obstetrics and Prenatal Medicine,

Erasmus University Medical Center Rotterdam,

Rotterdam, The Netherlands

A. Kloosterman

Department of Biological Traces,

Netherlands Forensic Institute,

The Hague, The Netherlands demonstrated until 2 months of age, and the $h P L$-specific RT-PCR assay applied was highly sensitive with reliable detection from down to $0.25 \mathrm{~cm}^{2}$ dried bloodstain. We therefore suggest $h P L$-specific RT-PCR as a new molecular tool for forensic pregnancy diagnostics from dried blood stains. Moreover, our results indicate that the time-wise reverse expression of $h P L$ and $\beta h C G$ during pregnancy may allow an RT-PCR-based estimation of the gestational age from blood stains, adding to the value of forensic pregnancy diagnosis for crime scene investigations.

Keywords mRNA markers · Pregnancy · Maternal blood · Forensic $\cdot h P L \cdot \beta h C G$

\section{Introduction}

The diagnosis of pregnancy from forensic bloodstains can be useful in cases of infanticide, criminal abortions and for missing person identification. However, this task is challenged by the usually small amounts of potentially degraded biological material found at crime scenes that often is of unknown age. Pregnancy diagnostics from bloodstains has been demonstrated via immunodetection of pregnancyspecific proteins [1], but the sensitivities achievable by this approach [2] limit its application in a forensic context where often minute dried bloodstains have to be investigated. Consequently, there is a need for more sensitive techniques to detect pregnancy-specific biomarkers from small bloodstains found at crime scenes. Research in prenatal diagnostics using reverse transcriptase polymerase chain reaction (RT-PCR) has discovered the presence of various placentaderived mRNA species in maternal plasma [3-6] that are reported to be remarkably stable in peripheral blood [7] and are rapidly cleared from circulation post-partum [3]. Also, 
unlike previously employed immunodetection methods, RTPCR diagnostics can be designed to be human-specific providing advantages in mixed human-animal samples where protein-based assays may produce false positive results. These characteristics make pregnancy-specific mRNA markers analyzed by RT-PCR technology a logical choice for the development of a pregnancy diagnostic test for forensic applications. We, therefore, tested by means of RT-PCR technology 11 genes previously reported with pregnancyspecific expression [3-6] and evaluated, for those with confirmed pregnancy-specific expression in whole blood stains, the in vitro time-wise stability of the transcripts as well as the RT-PCR sensitivity.

\section{Materials and methods}

\section{Sample collection}

To test for pregnancy-specific expression, we used blood samples from healthy volunteers with singleton pregnancies recruited from staff members of the Erasmus University Medical Center Rotterdam and the Netherlands Forensic Institute with informed consent. The samples were collected with sterile cotton swabs after a finger prick and stored at $-80^{\circ} \mathrm{C}$ or in RNAlater solution (Applied Biosystems, Nieuwerkerk aan de IJssel, the Netherlands) until RNA isolation. The gestational age of these female volunteers ranged from the 11.3th to the 37 th week of pregnancy (the digit behind the dot refers to extra days of the week indicated before the dot). One swab or $200 \mu \mathrm{l}$ of blood was used for RNA isolation. Saliva samples were collected from four pregnant volunteers in the 11.3th, 18th, 21st, and 37 th weeks of gestation; two cotton swabs fully soaked with fluid were used for RNA isolation. To determine the relationship between gene expression and gestation time, blood samples from women with singleton pregnancies were provided by the Department of Obstetrics and Gynecology of the Erasmus University Medical Center in Rotterdam. These samples had been collected by venipuncture during 2006 and 2007 in EDTA-containing tubes, stored at $-80^{\circ} \mathrm{C}$ and $200 \mu \mathrm{l}$ of blood was used for RNA isolation, which was performed in duplicate. The gestational age of these female volunteers ranged from the 8.2th to the 20.1 th week of pregnancy. In vitro time-wise stability of the transcripts was tested on blood sampled obtained with informed consent from three healthy volunteers who were in the 11.1th, 27.5th, and 30.5th weeks of pregnancy, respectively. For this, approximately $4 \mathrm{ml}$ of blood was drawn by venipuncture immediately processed to avoid clotting: $2 \mathrm{ml}$ of each blood sample was spread over a $56.7 \mathrm{~cm}^{2}$ piece of Whatman 3-MM filter paper placed in an uncovered Petri dish. The rest of the sample was absorbed with sterile cotton swabs. The filter paper and swabs were stored at ambient temperature in dust-free non-humid conditions and were subjected to normal daylight conditions. RNA from these samples was isolated after different time intervals; $1,7,14,28$, and 56 days. At each time point, RNA was extracted from one blood swab, as well as 1.0, 0.5, 0.25, $0.1,0.04$, and $0.01 \mathrm{~cm}^{2}$ pieces of blood-soaked and dried filter paper, which correspond to approximately 200.0, 35.3, $17.7,8.8,3.5,1.4$, and $0.4 \mu \mathrm{l}$ of spotted blood, respectively.

RNA isolation, cDNA synthesis and quantitative RT-PCR

Total RNA isolation was performed with the RNeasy Plus Mini kit (Qiagen Benelux B.V., Venlo, the Netherlands) according to the manufacturer's instructions with some modifications. These included the incubation of the cotton swabs and the pieces of filter paper in RLT buffer for $1 \mathrm{~h}$ at $4^{\circ} \mathrm{C}$ followed by acid phenol/chloroform extraction prior to the loading of whole blood lysate onto the RNeasy spin columns. This purification step significantly increased RNA yield and purity (data not shown). RNA extracts were treated with DNase using the Turbo DNA-free kit by Ambion (Applied Biosystems) to remove genomic DNA contamination. The quality and quantity of RNA was assessed with a NanoDrop ND-1000 spectrophotometer (Thermo Scientific, Wilmington, DE). For the RNA samples designated for the pregnancy specificity determination, cDNA synthesis was carried out with the Transcriptor First Strand cDNA Synthesis kit (Roche Diagnostics Nederland B.V., Almere, the Netherlands). RNA extracts designated for the gestation time applicability and sensitivity studies were reverse transcribed with the Revertaid H Minus First Strand cDNA Synthesis Kit (Fermentas, St. Leon-Rot, Germany) with a combination of random hexamer and oligo $(\mathrm{dT})_{18}$ primers. Real-time PCR reactions were performed in $15 \mu \mathrm{l}$ reaction volumes, in duplicate, on an ABI 7300 PCR machine (Applied Biosystems). The PCR reactions for $G A P D H$ and $\beta$-actin genes used as endogenous controls were performed with the FastStart Universal SYBR Green master mix (Roche Diagnostics Nederland B.V., the Netherlands) using previously described primer sequences $[8,9]$ and the following cycling parameters during PCR: initial denaturation at $95^{\circ} \mathrm{C}$ for $10 \mathrm{~min}$, followed by 50 cycles of denaturation at $95^{\circ} \mathrm{C}$ for $15 \mathrm{~s}$, and a final annealing-elongation at $60^{\circ} \mathrm{C}$ for $60 \mathrm{~s}$. The same SYBR Green PCR kit was used for the testing of the pregnancy specificity and the sensitivity of detection of the markers described in Oudejans et al. [4] (LOC90625 and CSH1 genes) and Go et al. [5] (ZDHHC1, GCM1, PSG9, and $P A P P A$ genes) using the primers and specific PCR conditions described in the original publications. RT-PCRs with hydrolysis probes were performed using the Maxima Probe qPCR Master Mix (Fermentas) with primer and 
probe sequences according to $\mathrm{Ng}$ et al. [3] for $h P L$ and $\beta h C G$ genes and Tsui et al. [6] for TFPI2, Kissl, and PLAC1 genes. All primers used in the study were either cDNA-specific or generated larger amplicons from genomic DNA template allowing a clear differentiation between products derived from mRNA and from genomic DNA. Agarose gel electrophoresis was performed to confirm the specificity of all amplifications.

\section{Results and discussion}

Pregnancy specificity of mRNA markers

Whole-blood RT-PCR analysis of 11 genes with previously reported pregnancy-specific expression pattern (LOC90625, CSH1, ZDHHC1, GCM1, PSG9, PAPPA, hPL, $\beta h C G$, TFPI2, Kiss 1, and PLAC1 [3-6]) performed in four samples of different pregnant women as well as in four samples of different non-pregnant female controls and in four male control samples provided confirmation for two transcripts, the human placental lactogen $(h P L)$ and the beta subunit of human chorionic gonadotropin $(\beta h C G)$. The remaining nine genes showed no pregnancy-specific expression pattern by analyzing whole blood. $H P L$ and $\beta h C G$ were also tested in samples of additional 4 different pregnant women; $h P L$ was detected in all eight samples from pregnant volunteers (Fig. 1), while $\beta h C G$ was detected in three of them. None of the control samples from males and non-pregnant females tested positive for any of the two transcripts. $H P L$ and $\beta h C G$ RT-PCR was also performed on saliva samples from four pregnant volunteers, and no amplification was detected (data not shown). This finding confirms expectations since $h P L$ and $\beta h C G$ expression is restricted to the placenta [10] allowing mRNA amplification from maternal blood but not from other body fluids of pregnant women that usually contain no blood. The use of whole blood in

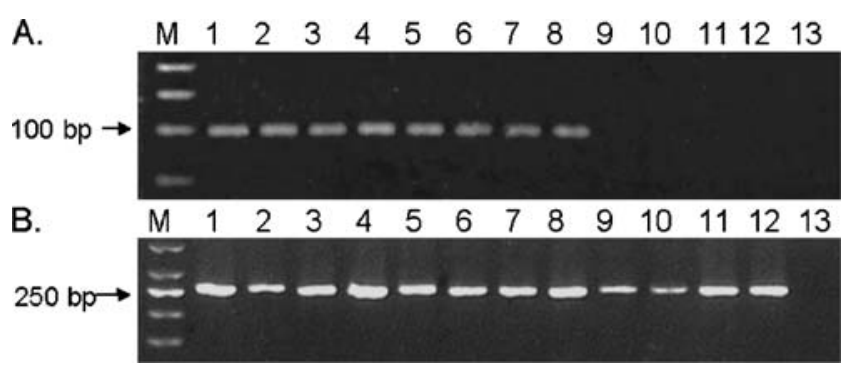

Fig. 1 Agarose gel electrophoresis with ethidium bromide staining of RT-PCR reactions with RNA from whole blood of eight different pregnant women in their 13th until 37th week of pregnancy (lanes 1-8) and four different non-pregnant women (lanes 9-12). a $h P L$ mRNA amplifications (expected product size: $97 \mathrm{bp}$ ). b $G A P D H$ mRNA amplifications (expected product size: $260 \mathrm{bp}$ ). Lane $M$ corresponds to a 50-base pair molecular size ladder and lane 13 corresponds to no template control reactions our study might serve as a possible explanation why the nine genes that showed pregnancy-specific expression in previous plasma-based studies [4-6] did not reveal pregnancy specificity in our study: cell components of whole blood could contribute to the overall expression profile and may have caused nonspecific amplification as observed here.

Time window of $h P L$ and $\beta h C G$ mRNA detection

To investigate the time window of $h P L$ and $\beta h C G$ gene expression throughout the pregnancy, we tested 25 additional samples from women in their 8.2th to 20.1th gestation week (Electronic Supplementary Material, Table S1). Together with the eight samples mentioned in the previous section, we detected $h P L$ in all 33 samples tested from women in their 8.2 th to 37 th gestation weeks, whereas $\beta h C G$ mRNA was successfully amplified from 24 of the 33 samples tested. Notably, $\beta h C G$ mRNA was not detected beyond the 20.1th week of pregnancy in the samples tested. These 25 samples were also used for reproducibility testing; for $h P L$, positive results were obtained for all technical replicates $(100 \%$ reproducibility), whereas the technical reproducibility of the $\beta h C G$ RT-PCR assay was $100 \%$ only with samples from women until the 10.1th week of pregnancy but decreased in samples from women of later gestational age (Electronic Supplementary Material, Table S1). These results are in line with previous reports $[3,10]$ showing that the concentration throughout the pregnancy of $h P L$ mRNA in maternal blood is higher relative to $\beta h C G$ and that the level of $\beta h C G$ mRNA decreases significantly after early pregnancy down to a few copies per milliliter of blood at late gestation age. The mRNA of both endogenous controls used (GAPDH and $\beta$ actin) was detected in all 33 samples of pregnant volunteers as well as in samples from males and from non-pregnant females without major quantitative differences (see Fig. 1 for $G A P D H$ ) demonstrating successful cDNA synthesis from the total RNA extracts and confirming previous reports of pregnancy-independent expression [3, 11]. Given that the amount of $h P L$ mRNA in maternal blood increases as the pregnancy progresses $[3,10]$, one can conclude that $h P L$ mRNA will be detectable at older gestation age than the 37th week available to us and until full term. The earliest point in the gestation period allowing the detection of $h P L$ and $\beta h C G$ mRNA is expected to be before the 8.2th week available to us; according to Okazaki et al. [10], both transcripts are detectable in samples from women of the sixth week of gestation.

Sensitivity of $h P L$ and $\beta h C G$ mRNA assays

Because the concentrations of $h P L$ mRNA increases with gestation time reaching the peak at the third trimester $[3$, 
10], we chose samples from the latest gestation period for which sufficient material was available to us (two donors in the 27.5th and the 30.5th week of gestation) to determine the smallest amount of dried blood required for reliable RTPCR detection of $h P L$ mRNA. We revealed that $h P L$ amplification is possible from as little as $0.25 \mathrm{~cm}^{2}$ dried bloodstain (corresponding to $8.8 \mu \mathrm{l}$ of liquid blood or $\sim 50$ ng total RNA). This approximately corresponds to the smallest reported amount from which the $h P L$ protein was detected previously by immunodetection [1]. Since the amount of $\beta h C G$ mRNA is maximal at the first trimester [3, 10], we used a sample from the earliest gestation period for which sufficient material was available to us (11.1th week) to determine the RT-PCR sensitivity limits for $\beta h C G$ mRNA detection. We found that reproducible $\beta h C G$ amplification could not be achieved by using less material than one standard blood-soaked dried cotton swab corresponding to about $200 \mu \mathrm{l}$ of liquid blood (or 200$1,000 \mathrm{ng}$ total RNA as extracted from dried blood). This is in line with $100 \%$ reproducibility of $\beta h C G$-specific RTPCR in samples from women in their 8.2th-10.1th week obtained from $200 \mu \mathrm{l}$ of liquid blood (or 500-2,000 ng total RNA; Electronic Supplementary Material, Table S1). However, we are aware that the gestation period of the sample available to us for this experiment does not correspond to the period of maximal $\beta h C G$ expression $[3$, 10]. The relatively high detection limit for $\beta h C G$ using the RT-PCR assay applied and the fact that the $\beta h C G$ transcript is not detectable throughout the entire gestational age limits the use of $\beta h C G$ mRNA to detect pregnancy from forensic blood stains (but see last chapter).
Time-wise stability of $h P L$ mRNA

The $C t$ values for $G A P D H, \beta$-actin, and $h P L$ PCR amplifications of the RNA extracted from the bloodstains obtained from women in the 27.5th and 30.5th weeks of pregnancy did not demonstrate any regular trend of change depending on the time of sample storage from 1 to 56 days, although increased variation of $C t$ values in the dried samples in comparison to the fresh ones was observed, as might be expected (data not shown). However, we were able to successfully detect $h P L$ mRNA in dried bloodstains after at least 56 days of stain exposure at room temperature. Although mRNA is generally believed to be highly unstable and prone to degradation due to the action of ribonucleases and environmental factors such as $\mathrm{pH}$, UV light, and moisture [12], it has been shown recently that some RNA markers might be exceptionally stable $[13,14]$. Therefore, our results of about 2 months in vitro stability indicate that sample degradation is not a strongly limiting factor for forensic pregnancy diagnostics, at least when using $h P L$ mRNA as marker and considering the environmental conditions applied.

Relationship between $h P L$ and $\beta h C G$ expression with gestation time

Initiated by the observed differences in the expression pattern between $h P L$ and $\beta h C G$ in samples of different gestation age, we were interested in studying the relationship between both transcripts throughout the pregnancy period in more detail. Using the RT-PCR data obtained
Fig. 2 Scatter plot of the average threshold cycle $(C t)$ value differences between $\beta h C G$ and $h P L$ mRNA amplifications of a given sample in relation to the gestational age of the sample donor. Only the samples with more than $50 \%$ of positive amplifications across all technical replicates were used to calculate the mean $C t$ and produce the scatter plot (see Electronic Supplementary Material, Table S1 for details)

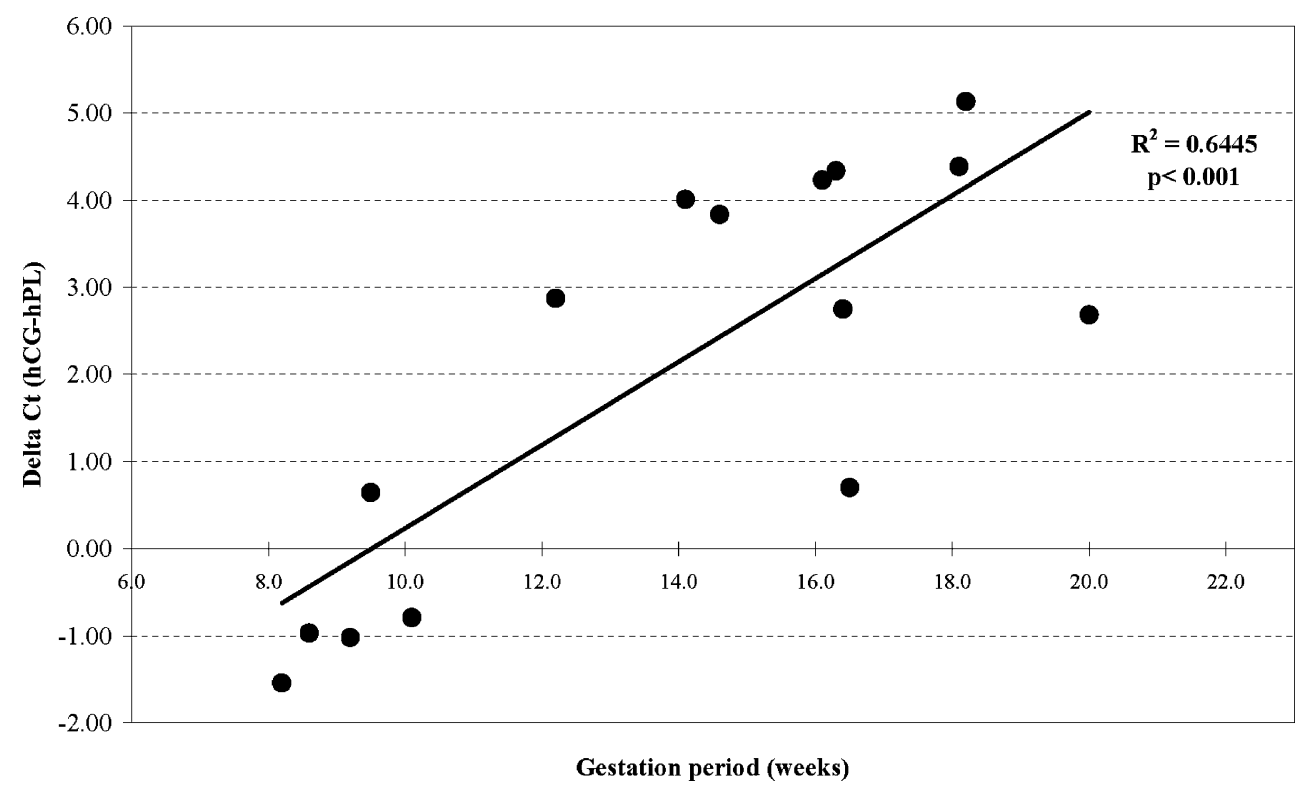


from the $h P L$ and $\beta h C G$ assays in samples from women in their 8.2th to 20.1th gestation week (Electronic Supplementary Material, Table S1), a statistically significant positive correlation $\left(R^{2}=0.6445, p<0.001\right)$ was observed between the threshold cycle $(C t)$ value differences of $\beta h C G$ and $h P L$ and the gestational age of the sample donor (Fig. 2). Even though $\beta h C G$ mRNA cannot be detected throughout the entire period of a normal pregnancy, our data indicate that when combined with the detection of $h P L$ mRNA, it may become useful in predicting gestational age from a forensic bloodstain that originated from a pregnant woman. However, the success of such additional application will depend on future improvements of the $\beta h C G$ RT-PCR assay, which may be achievable by further optimizing the three main steps of the RT-PCR approach: RNA extraction, cDNA synthesis, and PCR amplification and using samples from the complete pregnancy period.

\section{Conclusions}

Our data demonstrate that RT-PCR allows the detection of pregnancy-specific $h P L$ and $\beta h C G$ mRNA transcripts from small blood stains. The $h P L$-specific RT-PCR assay applied was sensitive enough to reliably detect $h P L$ transcripts throughout the pregnancy, and from as little as $0.25 \mathrm{~cm}^{2}$ of dried bloodstain. Furthermore, the $h P L$ transcript was shown to be stable enough for successful amplification from dried blood of up to 2 months of age, expectedly older. Therefore, we propose $h P L$-specific RTPCR as new molecular tool for pregnancy diagnostics from blood stains found at crime scenes. Moreover, our results indicate that a combined analysis of $h P L$ and $\beta h C G$ mRNA markers may allow estimating the gestational age of a woman from her blood stain left behind, potentially adding to the value of forensic pregnancy diagnosis for crime scene investigations.

Acknowledgements We are grateful to all volunteers who provided samples for this study. We thank Kaye Ballantyne for useful comments on the manuscript. This study was supported by funds from the Netherlands Forensic Institute.
Open Access This article is distributed under the terms of the Creative Commons Attribution Noncommercial License which permits any noncommercial use, distribution, and reproduction in any medium, provided the original author(s) and source are credited.

\section{References}

1. Vergote G, Heyndrickx B, Paredes M, Daels J (1991) Forensic determination of pregnancy hormones in human bloodstains. $\mathrm{J}$ Forensic Sci Soc 31:409-419

2. Vallejo G (1990) Human chorionic gonadotropin detection by means of enzyme immunoassay: a useful method in forensic pregnancy diagnosis in bloodstains. J Forensic Sci 35:293-300

3. Ng EK, Tsui NBY, Lau TK, Leung TN et al (2003) From the Cover: mRNA of placental origin is readily detectable in maternal plasma. Proc Natl Acad Sci U S A 100:4748-4753

4. Oudejans CBM, Go ATJJ, Visser A, Mulders MAM, Westerman BA, Blankenstein MA, van Vugt JMG (2003) Detection of chromosome 21-encoded mRNA of placental origin in maternal plasma. Clin Chem 49:1445-1449

5. Go AT, Visser A, Mulders MA, Blankenstein MA, Van Vugt JM, Oudejans CB (2004) Detection of placental transcription factor mRNA. Maternal Plasma Clin Chemistry 50:1413-1414

6. Tsui NBY, Chim SSC, Chiu RWK et al (2004) Systematic microarray based identification of placental mRNA in maternal plasma: towards non-invasive prenatal gene expression profiling. J Med Genet 41:461-467

7. Tsui NBY, Ng EKO, Lo YMD (2002) Stability of endogenous and added RNA in blood specimens, serum, and plasma. Clin Chem 48:1647-1653

8. Harper LV, Hilton AC, Jones AF (2003) RT-PCR for the pseudogene-free amplification of the glyceraldehyde-3-phosphate dehydrogenase gene (gapd). Mol Cel Probes 17:261-265

9. West JA, Park IH, Daley GQ, Geijsen N (2006) In vitro generation of germ cells from murine embryonic stem cells. Nat Protocols 1:2026-2036

10. Okazaki S, Sekizawa A, Purwosunu Y, Iwasaki M, Farina A, Okai T (2006) Measurement of mRNA of trophoblast-specific genes in cellular and plasma components of maternal blood. J Med Genet 43:e47

11. Murthi P, Fitzpatrick E, Borg AJ et al (2008) GAPDH, 18S rRNA and YWHAZ are suitable endogenous reference genes for relative gene expression studies in placental tissues from human idiopathic fetal growth restriction. Placenta 29(9):798-801

12. van Hoof A, Parker R (2002) Messenger RNA degradation: beginning at the end. Curr Biol 12:R285-R287

13. Heinrich M, Lutz-Bonengel S, Matt K, Schmidt U (2007) Real-time PCR detection of five different "endogenous control gene" transcripts in forensic autopsy material. Forensic Sci Int: Genetics 1:163-169

14. Zubakov D, Kokshoorn M, Kloosterman A, Kayser M (2009) New markers for old stains: stable mRNA markers for blood and saliva identification from up to 16-year-old stains. Int J Legal Med 123(1):71-74. doi:10.1007/s00414-008-0249-z 